Relations industrielles

Industrial Relations

\title{
L'évaluation des tâches
}

Job Evaluation

\section{Jean-Paul Deschênes}

Volume 16, numéro 2, avril 1961

URI : https://id.erudit.org/iderudit/1021798ar

DOI : https://doi.org/10.7202/1021798ar

Aller au sommaire du numéro

\section{Éditeur(s)}

Département des relations industrielles de l’Université Laval

\section{ISSN}

0034-379X (imprimé)

1703-8138 (numérique)

Découvrir la revue

Citer cet article

Deschênes, J.-P. (1961). L'évaluation des tâches. Relations industrielles / Industrial Relations, 16(2), 145-159. https://doi.org/10.7202/1021798ar
Résumé de l'article

Quoique l'évaluation des tâches soit une technique de plus en plus utilisée dans nos entreprises, il n'en demeure pas moins qu'elle est souvent peu comprise de ceux qui l'appliquent, la défendent ou la combattent. De plus, bien des systèmes ont mal fonctionné ou tout simplement échoué parce qu'on attendait d'eux des résultats qu'ils ne pouvaient ou ne devaient pas produire.
Tous droits réservés @ Département des relations industrielles de l’Université Laval, 1961
Ce document est protégé par la loi sur le droit d'auteur. L'utilisation des services d'Érudit (y compris la reproduction) est assujettie à sa politique d'utilisation que vous pouvez consulter en ligne.

https://apropos.erudit.org/fr/usagers/politique-dutilisation/ 


\title{
L'évaluation des tâches
}

\author{
Jean-Paul Deschênes
}

Quoique lévaluation des tâches soit une technique de plus en plus utilisée dans nos entreprises, il n'en demeure pas moins qu'elle est souvent peu comprise de ceux qui lappliquent, la défendent ou la combattent. De plus, bien des systèmes ont mal fonctionné ou tout simplement échoué parce quion attendait d'eux des résultats qu'ils ne pouvaient ou ne devaient pas produire.

Cet article n'a pas pour but de présenter une méthode idéale d'évaluation ni une procédure infaillible dans l'élaboration et l'application d'un système d'évaluation, mais tout simplement de replacer l'évaluation des tâches dans son contexte réel, en soulignant au passage ses avantages et ses faiblesses, dans l'espoir de contrebalancer quelque peu ces publications rédigées d'une part par des «vendeurs 》 pour qui l'évaluation est une panacée à tous les problèmes industriels, et, d'autre part, par des pseudo artistes de la négociation pour qui la détermination d'une structure des salaires à coup de poing sur la table est une source de satisfaction et de prestige.

Nous verrons donc dans les lignes qui suivent ce qu'est l'évaluation des tâches, la procédure suivie dans l'élaboration et l'application d'un système d'évaluation, celui des points ${ }^{1}$, ainsi que les moyens à prendre pour maintenir à date une structure des salaires.

\section{Ce qu'est l'évaluation des tâches}

L'évaluation des tâches est une technique objective qui permet de hiérarchiser les tàches, dans un groupe donné,

DESCHENES, JeAn-Paul, B.A., Maître en Sciences Sociales (Laval), Master of Science in Engineering (The Johns Hopkins University), membre de l'American Institute of Industrial Engineers, professeur au département des relations industrielles, Université Laval.

(1) Il existe d'autres méthodes d'évaluation que celle des points. Ce sont le rangement, la méthode des classes et la comparaison des facteurs. Dans cet article, je discute surtout de la méthode des points à cause de son usage très répandu. 
selon leur importance relative. L'évaluation proprement dite se termine lorsqu'on a assigné un certain nombre de points à chacune des tâches du groupe qu'on avait à évaluer.

Les difficultés que l'on rencontre ordinairement dans l'application et l'acceptation d'un système d'évaluation ne viennent souvent que de l'incompréhension que l'on a du système, et une erreur commune est de croire qu'une augmentation générale des salaires doit résulter de son application.

RAISON MOTIVANT UNE Évaluation

Pour bien illustrer cette affirmation que l'évaluation des taches se termine avec l'attribution de points aux tâches du groupe évalué, voyons des raisons qui motivent une évaluation des tâches.

Si les tâches n'ont jamais été évaluées de façon rationnelle (c'està-dire selon une méthode appropriée reconnue), il arrive que, involontairement ou non, des injustices se produisent et des tâches moins importantes sont les premières dans la hiérarchie des tâches et reçoivent une rémunération relative trop grande.

D'autre part, si les tâches ont déjà été évaluées, il arrive, avec le passage des mois et des années, que les méthodes de production évoluent, la machinerie désuète fait place à une autre plus rapide et plus précise et que le produit lui-même subit des modifications, de sorte que le contenu et l'importance de la tâche s'en trouvent modifiés. Comme ces changements dans les tâches ne se réalisent pas également dans toutes les tâches, la hiérarchie établie lors de l'évaluation se trouve à un moment donné, à ne plus correspondre à la réalité ${ }^{2}$.

Dans ces deux cas, une évaluation des tâches s'impose, non pour accorder des augmentations de salaire, mais pour les hiérarchiser selon leur importance relative.

(2) Si au moins une fois par année on a pris soin de réévaluer selon le système en vigueur, les tâches qui ont été modifiées, il est évident que la hiérarchie actuelle correspond à la réalité, et qu'il n'est pas question d'établir un nouveau système d'évaluation. Mais il arrive que lorsqu'on n'a pas pris soin de faire cette réévaluation, les tâches modifiées sont tellement nombreuses au bout d'un certain temps, que c'est tout le système d'évaluation qu'il faut reprendre à neuf. 
Evaluation ET STRUCture Des SALAIRES

Quoique la détermination de la structure des salaires est la suite logique et une des raisons d'être de l'évaluation des tâches, elle en est toutefois indépendante et il est malheureux que dans la pratique, les membres du comité qui doit évaluer les tâches aient aussi à discuter de la structure des salaires. Ce problème de la façon de déterminer la nouvelle structure des salaires devrait être réglé avant même que ne débute l'évaluation.

Il ne devrait pas normalement avoir de longues discussions sur la structure des salaires à adopter, car il ne s'agit, en fait, que de redistribuer la somme totale des salaires, déjà versée au groupe évalué selon les résultats de la classification. La méthode des moindres-carrés donne ces résultats. Il en résulte fatalement qu'un groupe de tâches se retrouvera au-dessous de la ligne de régression, qu'un autre groupe sera au-dessus de cette ligne et que très peu de tâches tombent exactement sur la nouvelle courbe des salaires. Les tâches au-dessous de la courbe sont automatiquement ajustées tandis que celles au-dessus sont ordinairement «gelés》 jusqu’à ce que les individus accomplissant ces tâches soient remplacés par d'autres ou que les augmentations générales des salaires négociées aux renouvellements du contrat parviennent à les fixer sur la courbe.

L'application de la méthode des moindres-carrés peut causer certaines commotions chez les patrons ou les employés en faisant varier les taux de rémunération de la tâche minimum et de la tâche maximum dans la hiérarchie, et ces taux sont souvent des points de repère qu'aucune des parties n'est prête à modifier. Dans ce cas, une droite est tirée entre ces deux taux de rémunération, et les autres tâches sont distribuées entre ces deux taux selon les résultats de l'évaluation. Là encore, il y aura des tâches au-dessus et des tâches au-dessous de la courbe, et il faudra les traiter de la façon décrite plus haut.

En définitive, à cause de cette façon de traiter les tâches au-dessus de la courbe, la somme totale des salaires versée aux employés sera plus grande après l'application du système d'évaluation.

Evaluation DES TÂChES - SCIENCE OU TEChNiQUe

L'évaluation des tâches n'est évidemment pas une science. Elle est une méthode, un outil permettant de rendre le plus objectif possible 
le jugement d'un groupe de personnes qualifiées dans la détermination de la valeur relative des tâches, et la moyenne de plusieurs jugements (ou un compromis entre ces jugements) ne rend pas la méthode plus scientifique. Seulement dans la façon de pondérer les facteurs, il y a beaucoup d'arbitraire et peu de science. Dans cette perspective, on ne peut demander ni aux patrons ni aux employés d'accepter sans une saine discussion les résultats de l'évaluation, et on ne peut non plus dépouiller le système de toute flexibilité.

Il était mentionné un peu plus haut que la méthode des moindrescarrés ou celle du minimum et du maximum donnait une ligne droite à laquelle les tâches devaient s'ajuster selon le nombre de points qui leur sont attribués. En pratique, toutefois, le résultat n'est pas si simple. On pourra, en effet, avoir des tâches dont limportance relative déterminée par l'évaluation pourrait fort bien se justifier à l'intérieur de l'usine mais dont le salaire qui devrait leur être payé ne correspond pas du tout aux taux qui prévalent sur le marché du travail. On pourra aussi avoir des tâches dont limportance relative devrait être diminuée, mais étant donné le statut social attaché à ces tâches, ou pour d'autres raisons d'ordre psychologique ou sentimental, il est impossible de le faire.

Il faut donc que les parties en cause prévoient une certaine flexibilité au système pour permettre une discussion intelligente et éclairee, mais aussi une certaine rigidité afin d'éviter que pour des prétextes futiles ou des motifs personnels ou égoistes, on empire une situation qu'on voulait corriger.

\section{Evaluation des tÂChes Et aUgmentation des SAlaires}

Une fois que l'évaluation des tâches a accompli son rôle, celui de redistribuer équitablement les salaires selon une base rationnelle, le champ est ouvert aux négociations, soit qu'il s'agisse d'augmenter le salaire de la tâche minimum ou de la tâche maximum, soit d'établir des groupes de tâches à l'intérieur desquels les tâches reçoivent le même salaire (échelle du type «escalier»), soit de déterminer un minimum et un maximum pour chaque tâche. Ces problèmes sont toutefois indépendants de l'évaluation proprement dite et ils ne doivent en aucune façon être discuter en même temps que l'évaluation comme s'ils en faisaient partie. Ceci éviterait, comme il arrive parfois, qu'une nouvelle hiérarchie des tâches, déterminée de façon rationnelle et accep- 
table en principe par les deux parties, ne soit mise au rancart parce qu'on ne s'entend pas sur les augmentations à accorder.

En plaçant ainsi l'évaluation des tâches dans sa perspective réelle, c'est-à-dire qu'elle n'est ni un système de rémunération au rendement, ni un moyen de réduire le coût de production ou d'augmenter les rendements des travailleurs, ni une méthode pour accorder des augmentations de salaires, il est difficile de comprendre qu'on puisse prévoir, dans une clause d'un contrat collectif, des montants appréciables « audelà des salaires actuels, pour bâtir un plan d'évaluation des tâches... $\gg^{3}$.

Il n'y a rien qui empêche les parties de négocier des nouveaux taux de salaire durant l'élaboration d'un système d'évaluation des tâches. Il ne s'agira, une fois l'évaluation complétée, que d'utiliser les nouveaux taux au lieu des anciens pour déterminer la structure des salaires.

\section{Les étapes dans l'élaboration d'un plan d'évaluation}

Voici succintement les différentes étapes à suivre dans l'élaboration d'un système d'évaluation des tâches:

1- Collection des données permettant une description adéquate de la tâche.

2- La description proprement dite de la tâche.

3- Le choix d'un certain nombre de tâches-clés ou tâches-repères.

4 - Le choix d'un système d'évaluation.

5- L'évaluation proprement dite.

6- La détermination de la nouvelle structure des salaires.

Avant d'aller plus loin dans l'explication de ces différentes étapes, il serait bon de préciser la participation logique de chacune des parties en cause dans l'élaboration et l'application d'un système d'évaluation des tâches.

Si l'évaluation des tâches avait un caractère purement scientifique, elle ne serait évidemment pas une matière à négociation, et lune des parties pourrait appliquer seule le système. Il n'y aurait qu'un seul résultat possible et il ne s'agirait, pour l'autre partie, que de vérifier si des erreurs ne se seraient pas glissées dans l'une ou l'autre des étapes.

(3) Picard, Gérard, Evaluation des tâches, Service d'éducation du Syndicat des Employés du Commerce de Montréal, Inc., 1959, p. 22 . Voir aussi pp. 17 à 23. 
Malheureusement, sous sa forme actuelle, l'évaluation des tâches n'est pas scientifique et ne fait que permettre, entre les deux parties, une discussion objective et systématique sur l'importance relative des tâches et leurs taux de rémunération. Dans l'application d'un tel système, il y a des questions à débattre à chacune des étapes et il est difficile de la faire unilatéralement. Chacune des parties en cause doit participer à la discussion.

Il n'est toutefois pas recommandable que tous et chacun à l'intérieur de l'organisation ait son mot à dire dans la méthode à suivre dans chacune des étapes qui conduisent à une nouvelle hiérarchie des tâches. Seulement un comité de personnes compétentes en matière d'évaluation peut le faire efficacement. Par conséquent, dans une négociation, on peut déterminer le rôle du syndicat, soit qu'il fasse partie du comité d'évaluation à même titre que la compagnie, soit qu'il ne se réserve que le droit de vérifier chacune des étapes franchies par le comité avec pouvoir de soumettre les modifications qu'il juge à propos.

Que le comité soit mixte ou unilatéral, il est d'absolue nécessité que ce soit des personnes compétentes qui en fassent partie et que les autres intéressés s'en remettent entièrement à leurs délégués, au moins jusqu'à la phase où les tâches sont hiérarchisées selon le nombre des points qui leur ont été accordés. Si le syndicat n'exerce qu'un contrôle, il faut aussi que ce contrôle se fasse par des personnes compétentes. Autrement, il est fort probable que l'évaluation ne parviendra jamais à terme, et il pourra se passer des semaines et des mois avant qu'on ait pu s'entendre sur les tâches-clés ou qu'on ait pu établir si la pondération devrait être $50 \%$ des points ou tout simplement $12 \%$. Chacun se spécialise dans son métier, et une connaissance parfaite de travail d'opérateur de pelle mécanique ne donne pas nécessairement une connaissance infuse de l'évaluation des tâches.

\section{LA DESCRIPTION DES TÂCHES}

La description des tâches est la base même d'une saine évaluation. Il faut donc prendre toutes les dispositions pour que cette description soit une image de la réalité. Un questionnaire approprié, rempli par l'employé, est susceptible de donner tous les éléments nécessaires à la rédaction de la description. Le comité d'évaluation s'appliquera ensuite à décrire la tâche sous sa forme définitive et en faire ressortir les exigences. Une vérification de la description par l'employé, son supérieur 
et son délégué départemental s'impose afin que tous soient d'accord sur les fonctions de la tâche et que rien d'important ne soit oublié.

\section{LE CHOIX DES TÂCHES-CLÉS}

On appelle tâches-clés celles qui sont représentatives, stables, connues et faciles à décrire. Il est à remarquer que toutes les méthodes d'évaluation (rangement, méthodes des classes, méthode des points et comparaisons des facteurs) peuvent ou doivent faire appel à un certain nombre de tâches-clés pour faciliter l'évaluation du reste des tâches, mais le fait qu'une méthode, comme celle des points, utilise des tâchesclés ne la rend pas essentiellement différente d'une méthode des points qui n'en utilise pas. Il n'y a donc pas, à proprement parler, de «système des tâches de repère ${ }^{4}$.

Le choix des tâches de repère doit se faire par le comité d'évaluation et celui-ci ne doit pas hésiter à mettre de côté une tâche d'abord choisie qui ne rencontre pas les caractéristiques d'une tâche-clé. Il est de beaucoup préférable d'avoir 15 tâches-clés adéquates sur un total de 200 tâches que d'en avoir 60 mal comprises et mal définies.

\section{LE CHOIX DU SYSTÈME D’ÉVALUATION}

Le choix du système d'évaluation est le point le plus délicat de toute la technique d'évaluation. C'est ici que les discussions sont des plus longues et souvent aboutissent à une impasse.

\section{Les facteurs et les sous-facteurs.}

Le choix des facteurs repose sur la description des tâches-clés. Il faut que chacun des facteurs se retrouve dans chacune des tâches à des degrés divers. Les facteurs ordinairement utilisés dans la méthode des points sont la compétence professionnelle, la responsabilité, l'effort et les conditions de travail. Mais parce que la compétence professionnelle et la responsabilité, pour ne nommer que ces deux facteurs, peuvent

\footnotetext{
(4) Voir Picard, Gérard, opus cit., pp. 50-57. Dans cette brochure, monsieur Picard semble confondre le rôle des tâches-clés dans une méthode d'évaluation. Toutes les méthodes peuvent se réaliser sans l'utilisation des tâches-clés, mais la méthode de comparaison des facteurs peut plus difficilement s'en passer. Le rôle des tâches-clés est de faciliter l'évaluation de l'ensemble des tâches. La méthode que monsieur Picard appelle « système des tâches de repère > est un système de points utilisant un certain nombre de tâches-clés.
} 
se décomposer en divers éléments ou sous-facteurs (vg. la compétence professionnelle peut se décomposer en instruction, expérience, initiative et ingéniosité, etc...) les systèmes d'évaluation peuvent reposer sur 10 , 12,15 sous-facteurs ou même plus. Le but des sous-facteurs n'est pas de rendre un système plus complexe mais bien de faciliter l'évaluation.

Il n'y a aucune objection à ce qu'un système comprenne 25 ou 30 sous-facteurs, mais à la condition toutefois que chacun de ces sousfacteurs soit distinct des autres, c'est-à-dire qu'il représente un aspect du facteur qui n'a pas été couvert par d'autres sous-facteurs. Ordinairement, au-delà de 12 ou 15 sous-fecteurs, on commence à noter des chevauchements d'un sous-facteur à l'autre.

\section{La pondération des facteurs.}

Les facteurs mentionnés plus haut n'ont pas tous la même valeur dans l'entreprise où un système d'évaluation est mis en application. Par exemple, pour des employés de bureau, on accordera beaucoup d'importance au facteur compétence professionnelle et peu au facteur effort physique. Il s'agit donc, dans l'élaboration d'un système d'évaluation, de pondérer ces facteurs.

Le cas le plus typique de la différence dans la pondération des facteurs se trouve dans deux systèmes bien connus soit le N.E.M.A. (National Electrical Manufacturers' Association) et le C.W.S. (Cooperative Wage Study). Le N.E.M.A. accorde environ $50 \%$ des points à la compétence professionnelle et environ $20 \%$ à la responsabilité alors que le C.W.S. accorde environ $50 \%$ des points à la responsabilité et $25 \%$ à la compétence professionnelle.

Le pourcentage des points accordé à chacun des facteurs ne repose sur aucune base scientifique rigoureuse. On peut toutefois énoncer certains éléments qui sont susceptibles de préciser la valeur relative des facteurs dans un système d'évaluation.

D'abord, je crois qu'on peut affirmer que cette pondération repose partiellement sur une échelle des valeurs admise jusquà un certain degré dans une société, et correspondant à son stage d'évolution. Dans cette échelle des valeurs de notre société présente, la compétence professionnelle vaut plus que l'effort physique ou les conditions de travail. 
Un autre élément qui peut influencer la pondération des facteurs est le marché du travail où on retrouve moins d'individus qui peuvent satisfaire aux exigences professionnelles d'une tâche et beaucoup plus qui peuvent satisfaire aux exigences physiques de la même tâche.

Il y a enfin la nature des tâches à évaluer qui va définitivement influencer la pondération des facteurs. Par exemple, si le groupe à évaluer ne comprend que des tâches manuelles plus ou moins spécialisées, on pourrait difficilement hiérarchiser ces tâches si on utilisait un système accordant $10 \%$ des points à l'effort physique et $60 \%$ à la compétence professionnelle. La même difficulté surviendrait si on utilisait un système accordant $10 \%$ à la compétence professionnelle et $60 \%$ à l'effort physique pour évaluer un groupe d'hommes de métier. Dans un cas comme dans l'autre, le nombre de points alloués pour évaluer ces tâches selon leurs caractéristiques propres ne serait pas suffisemment grand pour établir une différence marquée entre les tâches. A partir de ces observations, on ne peut que conclure qu'il est difficile de se prononcer sur la supériorité d'un système de points sur un autre sans connaître le groupe de tâches auquel il s'applique.

Nous avons mentionné plus haut que deux systèmes, le N.E.M.A. et le C.W.S. différaient passablement dans leur pondération de deux facteurs, la compétence professionnelle et la responsabilité, alors que pour les deux autres facteurs, conditions de travail et effort physique, la pondération était sensiblement la même. Faut-il en conclure que les mêmes tâches, évaluées selon ces deux systèmes, seraient ordonnées d'un façon totalement différente? Malheureusement les recherches sur ce problème sont peu nombreuses.

Toutefois, en dépit de ces différences apparentes entre les deux systèmes, il se peut que les résultats de chacun d'eux en regard d'un même groupe de tâches soient sensiblement les mêmes. Ceci serait dû au fait que dans la majorité des cas une tâche qui exige de grandes compétences professionnelles impose en même temps au candidat de lourdes responsabilités, alors qu'une autre tâche qui demande peu de compétences professionnelles exige peu ou pas de responsabilités. En d'autres mots, on constate dans la pratique que les compétences professionnelles et la responsabilité agissent dans le même sens, de sorte que, en prenant le cas extrême où l'un de ces deux facteurs serait enlevé et que l'autre se verrait attribuer les points de celui qui disparait, les résultats de l'évaluation demeureraient sensiblement les mêmes. Quant 
aux deux autres facteurs, conditions de travail et effort physique, ils agissent ordinairement en sens contraire aux deux premiers de sorte qu'il n'y a aucune substitution possible entre la compétence professionnelle et la responsabilité d'une part, et l'effort physique et les conditions de travail d'autre part.

Faut-il conclure de tout ceci que la compétence professionnelle ou la responsabilité devrait être éliminée d'un système d'évaluation? Evidemment non. D'abord parce que la parallèle entre les deux facteurs ne se réalise pas nécessairement dans toutes les tâches sans exception. Aussi, parce qu'en éliminant l'un de ces deux facteurs on négligerait un aspect important de la tâche, aspect à considérer lors de l'embauchage d'un candidat adéquat pour la tâche. Enfin, parce que, sur le plan psychologique, les employés se sentiraient frustrés de voir un aspect important de leur tâche sombrer dans l'oubli.

En définitive, la pondération des facteurs n'est pas d'importance capitale si les pourcentages accordés à chacun d'eux varient entre certaines limites logiques et acceptables. De même que dans la simplification des tâches, on constate qu'il n'y a pas de méthode de travail qui soit en elle-même la meilleure de toutes, de même dans l'évaluation des tâches, on constate qu'il n'y a pas de système qui en lui-même supplante tous les autres.

Le meilleur système d'évaluation est encore celui qui satisfait les deux parties intéressées et qui donne une hiérarchie des tâches-clés qui leur soit acceptable. Il peut même arriver qu'un système qui semble boiteux aux experts en évaluation donne des résultats satisfaisants aux parties en cause alors qu'un système, jugé idéal par une association de patrons ou par une fédération de syndicats ou d'unions et qu'on tente d'implanter dans plusieurs entreprises, donne des résultats pitoyables parce que les parties directement en cause ne l'acceptent pas.

Il ne faudrait pas conclure à la suite de ce qui précède que je favorise l'anarchie en matière d'évaluation. Je dis tout simplement que si un système a été préparé par des personnes compétentes et que ce système donne des résultats acceptables par les deux parties directement en cause, alors ce système est bon; l'outil a servi à la fin à laquelle on le destinait.

Quant aux sous-facteurs dont le rôle est de permettre d'analyser un facteur sous tous ses aspects, il est évident qu'eux aussi doivent être 
pondérés. Par conséquent, la majeure partie de la discussion que nous venons d'avoir sur les facteurs s'applique aussi aux sous-facteurs.

\section{Les degrés.}

Les degrés permettent de préciser davantage les sous-facteurs et de classifier les tâches selon leur importance quant au sous-facteur analysé. Par exemple, celui qui doit posséder une connaissance assez poussée de la mécanique pour accomplir son travail recevra plus de points sous ce sous-facteur qu'un autre qui ne doit connaître que les règles de la multiplication et de la division.

Trois règles principales gouvernent la détermination des degrés:

a) Ils doivent couvrir toute la gamme des tâches à évaluer.

b) Ils doivent être assez nombreux pour permettre une différentiation dans les tâches et pas trop nombreux non plus afin d'éviter des chevauchements d'un degré à l'autre.

c) Ils doivent être clairs, précis et sans ambiguité.

Les points accordés aux sous-facteurs sont distribués parmi les différents degrés de ce sous-facteur. Si par exemple le sous-facteur Instruction obtient 60 points et qu'on utilise 5 degrés pour expliciter ce sous-facteur, on pourra distribuer les points de la façon suivante:

Degré 1 - Jusqu'à la $7 \mathrm{e}$ année

Degré $2-2$ ans d'Ecole Technique

Degré $3-4$ ans d'Ecole Technique

Degré 4 - Maitrise

Degré 5 - Doctorat
- 12 points

-24 points

-36 points

-48 points

-60 points

N.B. Ceci n'est qu'un exemple très simplifié.

Cependant, au cours d'une évaluation, il arrive de constater que deux tâches sont classifiées dans un degré quelconque, disons le degré 2, mais que l'une est supérieure à l'autre. Ceci a amené certains spécialistes de l'évaluation à distribuer les points à l'intérieur des degrés de la façon suivante:
Degré 1 - de 0 à 12 points
Degré 2 - de 13 à 24 points
Degré 3 - de 25 à 36 points
Degré 4 - de 37 à 48 points
Degré 5 - de 49 à 60 points 
C'est alors que le comité de classification se perd en discussions pour établir si la tâche $X$ doit recevoir 1 ou 2 ou 3 points de plus que la tâche Y. Une formule intermédiaire qui a déjà été utilisée avec succès prévoit trois possibilités à l'intérieur d'un degré, soit un minimum, un standard et un maximum. Selon cette procédure, dans l'exemple donné plus haut, la répartition des points se ferait de la façon suivante:

\begin{tabular}{|c|c|c|c|}
\hline Degré 1 - & $\begin{array}{c}\text { Minimum } \\
4 \text { points }\end{array}$ & $\begin{array}{l}\text { Standard } \\
8 \text { points }\end{array}$ & $\begin{array}{c}\text { Maximum } \\
12 \text { points }\end{array}$ \\
\hline Degré 2 - & $16 “$ & 20 “ & $24 “$ \\
\hline Degré 3 - & 28 & 32 & 36 \\
\hline Degré $4-$ & 40 & 44 & 48 \\
\hline Degré 5 - & 52 & 56 “ & 60 \\
\hline
\end{tabular}

Il arrive souvent que des degrés soient demeurés inutilisés (surtout les plus élevés) une fois l'évaluation complétée, et cette situation doit être évitée. La principale raison est que la pondération initiale des facteurs (et des sous-facteurs) s'en trouve affectée. Par exemple, si on attribue 250 points sur 500 à la compétence professionnelle (soit $30 \%$ ), 50 points aux conditions de travail (soit $10 \%$ ) et 50 points à l'effort physique (soit $10 \%$ ) et que, par ailleurs, lors de l'évaluation, le maximum des points est 100 pour la compétence professionnelle, 100 pour la responsabilité, 50 pour les conditions de travail et 50 pour l'effort physique, la pondération initiale s'en trouve modifiée et les pondération finale est $331 / 3 \%$ pour la compétence professionnelle, $331 / 3 \%$ pour la responsabilité, $162 / 3 \%$ pour les conditions de travail et $162 / 3 \%$ pour l'effort physique.

Il arrive cependant que, intentionnellement, les responsables de l'élaboration d'un système d'évaluation incluent dans chacun des sousfacteurs un degré plus élevé que ceux réellement utilisés au cas où une tâche exigeant ce degré était créée à un moment donné. Si chaque sous-facteur contient ce degré supplémentaire et si l'accroissement des points accordés aux degrés suit une progression arithmétique, la pondération finale ne sera pas différente de la pondération originale.

\section{Evaluation Proprement dite}

Il n'y a pas lieu d'insister sur l'importance de cette étape. Toutes les autres étapes préparaient celle de l'évaluation proprement dite des tâches. Il y a cependant certains points sur lesquels jaimerais donner certains commentaires. 
La principale difficulté vient du fait que certains chefs sont portés à évaluer les tâches de leurs employés d'une façon trop rigide alors que d'autres considèrent que les tâches accomplies par leur personnel sont supérieures aux autres. Pour éviter ces écarts de jugement, il est bon d'avoir un comité d'évaluation composé d'environ 3 membres qui participent à l'évaluation de toutes les tâches. De plus, il est bon d'adjoindre à ce comité deux ou trois autres membres qui ne participent qu'à l'évaluation des tâches du groupe dont ils font partie. Ainsi, les membres permanents peuvent, jusqu'à un certain degré, voir à ce que les mêmes mesures soient appliquées à toutes les tâches, et les membres temporaires, du fait qu'ils connaissent mieux les tâches évaluées parce que faisant partie d'un même groupe, peuvent donner des détails utiles et fournir des explications pertinentes sur les tâches qui sont évaluées.

\section{REVISION ANNUELLE}

Une entreprise est essentiellement dynamique. Le produit est continuellement modifié et amélioré, et il en est ainsi des tâches. De plus, il n'est pas possible de prévoir les modifications que subiront les tâches dans leur hiérarchie parce que ces modifications reposent sur le progrès technologique, et l'évolution de progrès technologique ne peut être prévue parce qu'elle repose sur les découvertes scientifiques et sur la structure économique. Par conséquent, même si nous pouvons établir l'importance relative des tâches dans un groupe donné, cette hiérarchie tend à se désagréger dans une période de temps qui peut être assez courte.

Il faut donc périodiquement réviser le système d'évaluation en vigueur et l'adapter aux conditions présentes. Les entreprises qui revisent la hiérarchie des tâches le font ordinairement une fois par année.

Il est important que la révision annuelle de l'évaluation se fasse autant que possible par le même comité qui a établi le système. Quand on parle d'évaluation, on parle de jugement, et la façon de juger une tâche peut différer d'un individu à l'autre et d'un comité à l'autre. Il faut donc conserver autant que possible les conditions dans lesquelles la première évaluation a été réalisée, et la composition du comité de révision en est une.

Dans la révision, il s'agit non pas de réévaluer toutes les tâches, mais seulement celles qui ont subi des modifications au cours de l'année. Il faut donc que le comité ait en mains, lors de sa réunion, tous les documents et toutes les informations concernant les modifications dans 
les tâches. Pour ce faire, il s'agit de prévoir une formule spéciale où l'individu consignerait les modifications permanentes dans sa tâche. Cette formule, approuvée par le supérieur de l'individu et par le délégué départemental s'il y a lieu, permet de procéder à une nouvelle évaluation de la tâche modifiée en se basant sur des faits précis.

\section{Conclusion}

Comme il a été mentionné au début, cet article n'avait pas pour but de présenter au lecteur un système d'évaluation supérieur aux autres et susceptible de régler définitivement le problème complexe et épineux de la valeur relative des tâches dans une organisation. L'évaluation des tâches est une technique permettant de porter un jugement objectif sur la valeur d'une tâche en la comparant à d'autres tâches, et le système le meilleur dans une entreprise est celui qui donne satisfaction aux deux parties.

De plus, quelles qu'en soient les faiblesses, cette technique permet d'éviter, et c'est là son plus grand mérite, que des groupes plus puissants ne négocient des salaires que pour eux, qu'un chef syndical ne profite de sa position pour avoir un salaire plus élevé et que le patron ne rémunère davantage un ancien confrère de classe ou un membre d'un même club indépendemment de la fonction accomplie.

Que l'évaluation des tâches n'ait pas un caractère scientifique ct que les résultats n'aient pas une précision mathématique, il n'y a pas lieu de s'en désoler. On pourra avoir une évaluation à caractère scientifique lorsque l'homme agira comme une machine, lorsque ses pensées et ses actions s'orienteront dans une seule direction prévisible lorsqu'il sera placé dans des circonstances connues et prédéterminées. Mais à ce moment, ce ne sera plus un homme qui accomplira une tâche, mais un robot.

Que l'évaluation des tâches, comme l'étude des temps, n'ait pas un caractère scientifique, ceci indique que l'homme, même à l'usine, reste un homme intelligent et libre, garde sa dignité d'homme.

Ceci ne diminue en rien la valeur réelle d'un système d'évaluation des tâches bien compris et bien appliqué. Un tel système a pour but de réduire à un minimum l'arbitraire dans la détermination de la valeur relative des tâches et de permettre des discussions systématiques sur les tâches et leur rémunération. 


\section{JOB EVALUATION}

Job evaluation is an objective technique to establish the relative importance of jobs in a given group. The wage structure may be the result of the application of a job evaluation system, but such a system can serve many other purposes. Therefore, the wage structure itself is independant of the job evaluation technique and must be discussed outside the committee involved in the process of evaluating the jobs.

Job evaluation is a technique, not a science. It is a tool which permits an objective judgment on the importance of a job in relation with others. Therefore, both parties, management and employees, should participate in the process of evaluating jobs. Job evaluation, however, must be done by competent persons. If everybody in the organizations has a word to say on how the problem must be solved, there are great possibilities that the plan will never be applied because of deadlocks which will arise.

One main problem which has to be solved in designing a job evaluation system is the weight to be given to factors and sub-factors. There is important differences between plans, in particular between the C.W.S. and the N.E.M.A., when the former gives about $25 \%$ of the points to skill and $50 \%$ to responsibility and the latter gives $50 \%$ to skill and $20 \%$ to responsibility.

One can say that the weight gives to factors depends on the degree of evolution of the society, the labor market and first of all on the caracteristics of the jobs which are to be evaluated. As far as the C.W.S. and the N.E.M.A. are concerned, it is possible that their differences are only apparent as skill and responsibility work in the same direction, i.e. a job requiring great skill imposes also great responsibility on the incumbent, so that we can vary the weight between these two without influencing the final result.

The main advantage of a job evaluation system is to remunarate a job according to the requirements of the job and not to the individual himself, and to permit an objective judgment on the relative importance of jobs in a given group.

\section{RECHERCHES SOCIOGRAPHIQUES}

revue trimestrielle publiée par le Département de Sociologie de l'Université Laval, Québec, Canada.

Direction: JEAN-C. FALARDEAU et FERNAND DUMONT Secrétaire de la rédaction: Yves MARTIN

Sommaire du no 1, vol. II (janvier-mars 1961)

JaCques HenRipin

GÉrald Fortin et Louis-Marie Tremblay

Pierre Bélanger et ANDRÉ JUNEAU

LÉON DiON
L'inégalité sociale devant la mort: la mortinatalité et la mortalité infantile à Montréal. Attitudes à l'égard des occupations dans une paroisse agricole.

Les maîtres de l'enseignement primaire: étude socio-culturelle.

Le libéralisme du statu quo: le droit protecteur.

Situations de la recherche - Comptes rendus

Abonnement (un an, quatre numéros): Canada, \$5.00; autres pays: \$5.50. Administration et abonnenents: Les Presses Universitaires Laval, 28, rue Sainte-Famille, Québec 4, Canada. - Direction et rédaction: Département de Sociologie, Faculté des sciences sociales, Université Laval, Québec 4, Canada. 